AperTO - Archivio Istituzionale Open Access dell'Università di Torino

Late onset bipolar disorder and frontotemporal dementia with mutation in progranulin gene: a case report

This is a pre print version of the following article:

Original Citation:

Availability:

This version is available http://hdl.handle.net/2318/1659140

since 2018-01-25T11:34:24Z

Published version:

DOI:10.1080/21678421.2017.1339716

Terms of use:

Open Access

Anyone can freely access the full text of works made available as "Open Access". Works made available under a Creative Commons license can be used according to the terms and conditions of said license. Use of all other works requires consent of the right holder (author or publisher) if not exempted from copyright protection by the applicable law. 


\section{Late onset bipolar disorder and frontotemporal dementia with mutation in progranulin gene: a case report}

Elisa Rubino*11, Alessandro Vacca ${ }^{1}$, Salvatore Gallone ${ }^{2}$, Flora Govone ${ }^{1}$, Milena Zucca ${ }^{1}$, Annalisa Gai $^{1}$, Patrizia Ferrero ${ }^{2}$, Pierpaola Fenoglio ${ }^{2}$, Maria Teresa Giordana ${ }^{1,2}$, Innocenzo Rainero ${ }^{1,2}$

${ }^{1}$ Aging Brain and Memory Clinic, Department of Neuroscience "Rita Levi Montalcini”, University of Turin, Via Cherasco, 15, 10126 Turin, Italy

${ }^{2}$ Neurology I, Department of Neuroscience and Mental Health, AOU Città della Salute e della Scienza, via Cherasco 15, 10126 Turin, Italy

Keywords: bipolar disorder type 1, frontotemporal dementia, progranulin gene

*Corresponding author

Elisa Rubino

Department of Neuroscience "Rita Levi Montalcini”

University of Torino

Via Cherasco 15 - 10127 Torino

mail: elisa.rubino@unito.it

Phone: + 39-011-6334763

Fax: +39-011-6638510 


\begin{abstract}
Bipolar disorder (BD) is a chronic psychiatric illness characterized by fluctuation in mood state, with a relapsing and remitting course. Frontotemporal dementia (FTD) is a clinically and genetically heterogeneous syndrome, with the most frequent phenotype being behavioral variant frontotemporal dementia (bvFTD). Here, we report the case of an Italian male presenting with late onset bipolar disorder, that developed bvFTD over time, carrying a mutation in the GRN gene. Interestingly, the patient carried the c.1639 C>T variant in $G R N$ gene, resulting in a R547C substitution. Our case report further corroborates the notion that, in addition to FTD, progranulin may be involved in the neurobiology of bipolar disorder type 1, and suggests to screen patients with late onset bipolar disorder for GRN mutations.
\end{abstract}




\section{Introduction}

Bipolar disorder (BD) is a psychiatric illness characterized by fluctuation in mood state with a relapsing and remitting course. Genetic studies showed that BD is a complex disease, consisting of both common and rare susceptibility variants (1). Frontotemporal dementia (FTD) is a clinically heterogeneous syndrome. The most frequent phenotype is behavioral variant frontotemporal dementia (bvFTD), followed by semantic dementia, and progressive non-fluent aphasia. Three genes have been shown to be major causes of FTD: microtubule-associated protein tau (MAPT), progranulin (GRN), and C9ORF72 (2).

Here we report the case of an Italian male presenting with late onset bipolar disorder, that developed bvFTD carrying the R547C mutation in GRN gene.

\section{Case Report}

A 70 years old man was diagnosed with bipolar disorder type 1 at 55 years of age. He reported depressive episodes when he reached his thirties. The onset of maniacal symptoms was at 54 years, when the patient presented elevated mood, impulsiveness, and grandiose delusion. The patient was hospitalized in a psychiatric ward and a diagnosis of BD type 1 was made. Thence, a combined therapy with escitalopram and carbolithium was introduced with improvement of psychiatric symptoms. At 67 years of age, the patient was newly hospitalized for acute lithium intoxication (plasmatic range 1,89 mEq/l) with severe hypernatriemia and ipokaliemia. At discharge, he did not show any signs of cognitive impairment and he had a complete recover.

At 68 years, 15 years after the diagnosis of BD, the patient become less extrovert, and presented with indifference, and hyperphagia. The clinical picture progressively deteriorated and he became apathetic with lacking motivation, and retiring from social and leisure activities. The patient was 
admitted to our Aging Brain and Memory Clinic, Department of Neuroscience, University of Torino. Familial history revealed that the son was suffering by panic attacks, while the deceased mother and the maternal grandmother were affected by late onset unspecified dementia, suggesting an autosomal dominant inheritance. The neurological examination showed mild bilateral parkinsonian signs. The neuropsychological assessment revealed a mild cognitive impairment (MMSE was 22.4/30), deficit in selective attention, verbal memory, and executive dysfunction. Cranial CT evidenced asymmetrical brain ventricles and mild frontotemporal atrophy (Fig. 1A). CSF examination showed normal values of total Tau (152.6 pg/ml), posphorilated Tau $(34.7 \mathrm{pg} / \mathrm{ml})$, and A $\beta$-42 (805.6 pg/ml). Brain 18-FDG PET revealed marked hypometabolism in bilateral frontotemporal areas. A diagnosis of bvFTD was made according to Rascovsky et al. criteria (3). MAPT, GRN, C9ORF72 genes were sequenced and we did not detect neither mutations in MAPT nor expansions in C9ORF72. Interestingly, the genetic analysis revealed the c.1639 C>T variant in GRN gene, in exon 12, resulting in a R547C substitution. We considered the variant as a pathogenic mutation since a) at least three of the four in silico tools predicted the variant as pathogenic (Polyphen2, GVGD and SNAP predicted the variant to be deleterious, whereas SIFT classified it as neutral); b) the variant affects a highly evolutionary conserved aminoacid (Fig. 1B); c) the change was absent in genetic databases (ExAc, dbSNPs, and 1000 Genomes).

\section{Discussion}

Our case report confirms previous studies suggesting an association between bipolar disorder type 1 and progranulin mutations (4). In addition, we report the first association between BD type 1 and the genetic variant R547C, a mutation previously described in a bvFTD patient with parkinsonism and impulse control disorder (5). Due to the limitations of in silico prediction programs and the lack 
of additional family members to test for co-segregation, functional studies are suggested to further support the pathogenicity of the identified variant.

Notably, GRN polymorphisms have been shown to contribute to the susceptibility to BD type 1, and progranulin plasma concentrations are lowered in patients with BD (6). Progranulin is a pleiotropic protein widely expressed in the nervous system both during development and adulthood. Little is known about the normal function of the protein in CNS, apart from a role in inflammation, longterm neuronal survival, and neurodegeneration (7).

Psychiatric symptoms have been reported as initial manifestations of bvFTD, in particular in the genetic forms due to an expansion in C9ORF72 (up to 50\% of cases) (8), and to mutation in GRN (up to $25 \%$ of patients) (9). Our patient presented an affective disorder before the bvFTD diagnosis, and this may suggest that the BD might represent a preclinical phase preceding the onset of frontotemporal dementia.

In conclusion, our case report further corroborates the notion that progranulin may also be involved in the neurobiology of bipolar disorder type 1, and suggests to screen late-onset patients for $G R N$ mutations. 
Declaration of interest: The authors report no conflicts of interest.

Funding/support: None.

Acknowledgment: The authors would like to thank the patient and his family. 


\section{References}

1. Craddock N, Sklar P. Genetics of bipolar disorder. Lancet 2013;381:1654-62.

2. Benussi A, Padovani A, Borroni B. Phenotypic Heterogeneity of Monogenic Frontotemporal Dementia. Front Aging Neurosci 2015;7:171.

3. Rascovsky K, Hodges JR, Knopman D, Mendez MF, Kramer JH, Neuhaus J, et al. Sensitivity of revised diagnostic criteria for the behavioural variant of frontotemporal dementia. Brain 2011;134:2456-77.

4. Cerami C, Marcone A, Galimberti D, Villa C, Scarpini E, Cappa SF. From genotype to phenotype: two cases of genetic frontotemporal lobar degeneration with premorbid bipolar disorder. J Alzheimers Dis 2011;27:791-7.

5. Wong SH, Lecky BR, Steiger MJ. Parkinsonism and impulse control disorder: presentation of a new progranulin gene mutation. Mov Disord 2009;24:618-9.

6. Galimberti D, Prunas C, Paoli RA, Dell'Osso B, Fenoglio C, Villa C, et al. Progranulin gene variability influences the risk for bipolar I disorder, but not bipolar II disorder. Bipolar Disord 2014;16:769-72.

7. Cenik B, Sephton CF, Kutluk Cenik B, Herz J, Yu G. Progranulin: a proteolytically processed protein at the crossroads of inflammation and neurodegeneration. J Biol Chem 2012;287:32298306.

8. Galimberti D, Fenoglio C, Serpente M, Villa C, Bonsi R, Arighi A, et al. Autosomal dominant frontotemporal lobar degeneration due to the C9ORF72 hexanucleotide repeat expansion: late-onset psychotic clinical presentation. Biol Psychiatry 2013;74:384-91.

9. Lanata SC, Miller BL. The behavioural variant frontotemporal dementia (bvFTD) syndrome in psychiatry. J Neurol Neurosurg Psychiatry 2016;87:501-511. 